\title{
Sudden changes in spectrum of an echo cause a breakdown of the precedence effect
}

\author{
DANIEL D. MCCALL, RICHARD L. FREYMAN, and RACHEL K. CLIFTON \\ University of Massachusetts, Amherst, Massachusetts
}

\begin{abstract}
The effect of changing the frequency components of an echo relative to the sound source was examined in a two-choice discrimination task. Subjects sat in an anechoic chamber and discriminated the direction of the lag noise burst within a lead-lag pair presented over loudspeakers. The leading noise burst was broadband, and the lagging burst was either high- or low-pass filtered. On some conditions, this test burst pair was preceded by a conditioning train of burst pairs, which also had a broadband lead and either a high- or low-frequency lag. When the frequency content of the echo was held constant across the conditioning train and test burst pair, echo suppression that was built up during the repeating train was maintained for the test burst pair, shown by the subjects' poor performance in detecting the location of the lagging burst. By comparison, subjects had little difficulty in localizing the lagging burst when the frequency content of the echo changed between the conditioning train and the test burst, indicating that any buildup of suppression during the train was broken when the lagging burst's spectrum shifted. The data are consistent with an interpretation in which echo suppression is temporarily broken when listeners' built-up expectations about room acoustics are violated.
\end{abstract}

Sounds produced in an enclosed space produce reflections off surrounding surfaces. Given a brief delay (several milliseconds) between an original sound source and its reflection, a listener will not perceive the reflection as a separate sound source but instead will localize a single sound near the location of the leading sound source. This ability of the auditory system to suppress the perception of the direction of echoes has been termed the precedence effect (Wallach, Newman, \& Rosenzweig, 1949; Zurek, 1987).

Recent evidence has suggested that the precedence effect is not simply the result of a peripheral echo suppression mechanism but may also be mediated by more central, higher level perceptual processes (Blauert \& Col, 1992; Clifton \& Freyman, 1997; Clifton, Freyman, Litovsky, \& McCall, 1994; Hafter, Buell, \& Richards, 1988; Hartmann, 1997; Yost \& Guzman, 1996). In a recently developed model of spatial hearing, Blauert (1996) explicitly delineated a role for "top-down" processes. By this, he refers to a hypothesis-driven process that evaluates the bottom-up binaural input. At the perceptual level, the listener responds on the basis of auditory events and auditory images. These

This research was supported by Grant DC01625 from the National Institute on Deafness and Other Communication Disorders to R.L.F. and R.K.C., and by Research Scientist Award MH00332 from the National Institute of Health to R.K.C. D.D.M. received support from NIMH Training Grant MH16745. Some of the results were reported at the 127th Meeting of the Acoustical Society of America, June 1994 (Freyman, Clifton, \& McCall, 1994). The authors thank two anonymous reviewers for helpful comments and William Yost for suggesting a localization explanation for the difficulty that subjects had with the BL-BH condition. Correspondence should be addressed to R. K. Clifton, Department of Psychology, University of Massachusetts, Amherst, MA 01003 (e-mail: rachel@psych.umass.edu). perceptions are influenced by the listener's prior experiences, expectations, and even input from other modalities. Blauert (1996, pp. 409-422) reviews the literature and makes a case for the precedence effect as a good example of such top-down influences. Previously, Clifton et al. (1994) conceptualized the precedence effect as a decision-making process that is affected by ongoing auditory stimulation. When we describe the precedence effect as involving "decisions," we do not refer to a deliberate, conscious decision on the part of the listener to think about the sound. The process is rapid and not under conscious control. Here, it is well to remember that the precedence effect is an auditory illusion and, like most illusions, is not under voluntary control. You cannot "will yourself" to hear the echo. In precedence effect experiments, the listener may fail to detect the echo as a separate, audible sound, even though the echo may be delivered with intensity equal to the leading sound. Furthermore, the listener has been instructed that an echo is present on every trial, and their task is to discriminate or detect that echo. To understand the precedence effect is to understand the circumstances under which the listener sometimes succeeds in this task and sometimes does not.

In a series of papers, we have demonstrated that listeners develop expectations about their acoustic environment that influence their perception of sound sources and their associated reflections. Echo threshold, defined as the shortest delay between a sound source and its reflection that allows a listener to localize the lagging sound as a separate auditory event (Blauert, 1983, pp. 222-224), can be raised or lowered depending on the listener's experiences in a simulated acoustic environment. Echo threshold is raised when a listener is presented with ongoing, redundant information. For example, when a train of click- or noise-burst 


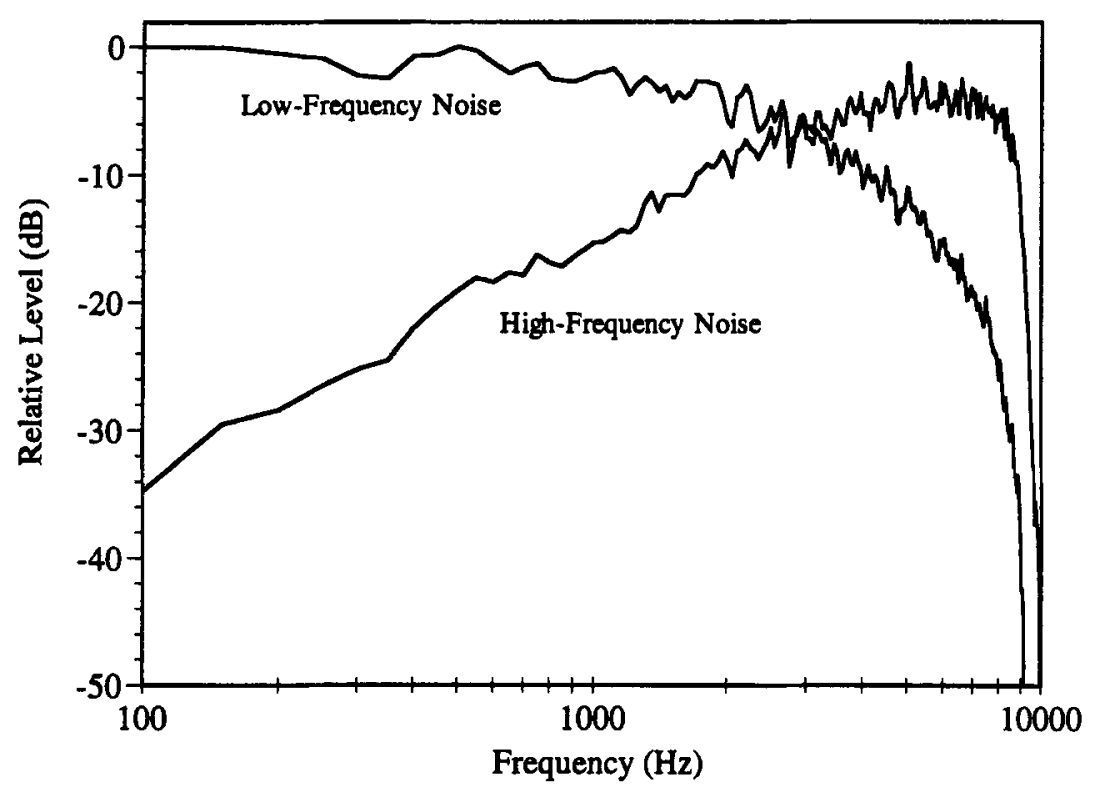

Figure 1. Frequency composition of the low-frequency emphasis and high-frequency emphasis echoes.

pairs is presented from two loudspeakers with one lagging the other by a few milliseconds, the listener will initially hear two separate sound sources. However, as the train of stimulation continues, echo suppression is built up that is, the lagging stimulus fades out, and a single image is localized at the leading loudspeaker (Clifton, 1987; Clifton \& Freyman, 1989; Thurlow \& Parks, 1961). We hypothesized that this buildup of echo suppression can be disrupted (shown by a lowering of echo threshold) if the stimulus and/or its echo is suddenly changed in a way that is improbable given the acoustic environment. One example of an improbable change is to suddenly switch the spatial location of the primary source and its echo during the train; this change causes a breakdown in echo suppression (Blauert \& Col, 1992; Clifton, 1987; Clifton \& Freyman, 1989). Similarly, echo threshold is lowered when the delay between lead and lag stimuli is suddenly changed in a manner that simulates the sudden movement of a reflective surface (Clifton et al., 1994). On the other hand, sudden changes that signify more probable, expected environmental cues do not cause echo suppression to break down. When the primary source and its identical echo simultaneously change in frequency or intensity (Clifton et al., 1994), or the source and its echo are shifted laterally in space (Yost \& Guzman, 1995), echo suppression is maintained. Such changes are probable in that they signify common occurrences in our environment (i.e., sounds are constantly changing in frequency, intensity, and location, and their associated echoes reflect these changes).

The present study examined this expectation hypothesis further by manipulating the amplitude of frequency components of the echo relative to the sound source. While a change in the spectrum of both leading and lagging sounds denotes a common environmental occurrence in which the sound source (e.g., a voice) is varying over time, sudden frequency changes in the echo alone indicate a dramatic change in the absorption qualities of a reflective surface, a bizarre and unusual circumstance. Such a change should result in a breakdown of echo suppression. In the present study, echo suppression was built up through repeated presentation of a broadband leading stimulus and a delayed echo that was either high- or low-pass filtered, simulating an acoustic environment in which a reflective surface absorbed some frequencies more than others. The spectrum of the echo was then suddenly changed, simulating an abrupt, unexpected change in the absorption qualities of the reflective surface. It was predicted that this change would cause echo suppression to break down, allowing subjects to hear the previously suppressed echo as a separate source.

\section{METHOD}

\section{Subjects}

Six normal-hearing adults participated. All had pure-tone air conduction thresholds less than or equal to $15 \mathrm{~dB} \mathrm{HL}$ at $0.25,0.5,1.0$, $2.0,3.0,4.0,6.0$, and $8.0 \mathrm{kHz}$, and all had no more than a $10-\mathrm{dB}$ discrepancy between the two ears at any frequency. One additional subject was screened and eliminated from the sample for failure to show any buldup of echo suppression.

\section{Stimuli and Apparatus}

The stimuli were 4-msec noise bursts, shaped with 2-msec linear rise/fall times, which had been sampled from three longer ( $400-\mathrm{msec})$ segments of computer-generated white noise. One segment was broadband, and the other two were the same broadband noise that had been either low-pass filtered with a cutoff frequency of $2 \mathrm{kHz}$ or high-pass filtered with a cutoff frequency of $4 \mathrm{kHz}$. The filters were single pole digital filters. Amplitude spectra of the noises were measured by presenting the $400-\mathrm{msec}$ segments continuously, without pause between repeated segments. Spectral analysis was performed on the electrical signal after an antialiasing filter $(8.5 \mathrm{kHz})$ using an HP 3569A analyzer to compute and average 100 fast Fourier transforms. Figure 1 shows the resulting spectra for the high- and low-frequency noises. 


\section{Lead Loudspeakers}

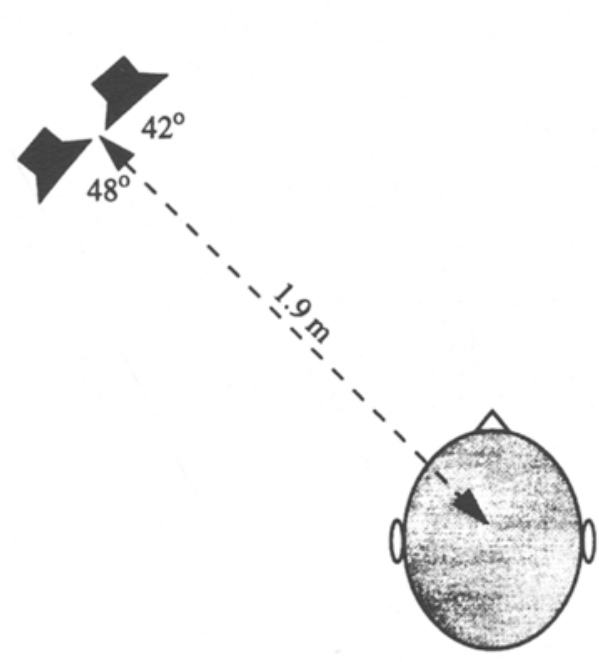

ANECHOIC CHAMBER
Lag Loudspeakers

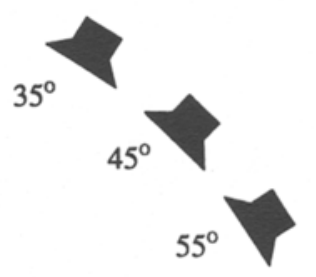

Figure 2. The loudspeaker configuration used. The leading stimulus was presented from one of the two left loudspeakers (varied randomly from trial to trial). On the test burst, the lagging stimulus was presented from one of the speakers at either $35^{\circ}$ or $55^{\circ}$ right of midline. On the conditions on which the test burst was preceded by a train of burst pairs, the lagging stimulus was presented from the loudspeaker at $45^{\circ}$ right and switched to $35^{\circ}$ or $55^{\circ}$ on the test burst.

Noise bursts were presented in lead-lag pairs, with the subject's response based on a decision about the final burst pair for each trial, called the test burst pair. For most conditions, this test burst pair was preceded by a conditioning train of 17 lead-lag burst pairs, presented at a rate of $8 / \mathrm{sec}$. A delay of $750 \mathrm{msec}$ separated the conditioning train from the test burst pair. To randomize sound from trial to trial, 10 tokens of noise were sampled from each of the three longer noises to serve in the test burst pairs, and an additional 85 tokens were sampled from each noise to construct five different trains of burst pairs at 17 bursts per train. Noise bursts were presented from two channels of a 16-bit D/A (TDT QDA2), with the delayed burst in the right channel. The outputs of the two channels were low-pass filtered at $8.5 \mathrm{kHz}$ (TTE J1390), attenuated (TDT PAT 1), amplified (NAD 2100), multiplexed (TDT AMUX1), and connected to a set of loudspeakers (Realistic Minimus 7), matched for overall level.

The subject sat in a chair at the center of an anechoic chamber $(4.9 \times 4.1 \times 3.1 \mathrm{~m})$ whose floor, ceiling, and walls were covered with $0.72-\mathrm{m}$ foam wedges. Five loudspeakers were located at $42^{\circ}$ and $48^{\circ}$ left and $35^{\circ}, 45^{\circ}$, and $55^{\circ}$ right of midline at a distance of $1.9 \mathrm{~m}$ from the subject's head (see Figure 2). The center of the loudspeakers was $1.04 \mathrm{~m}$ above the wire mesh floor of the chamber, the approximate height of the typical subject's ears while seated in the charr. Stimulus level was measured using a microphone placed at the position of the center of the subject's head and by presenting trains of noise bursts at $8 / \mathrm{sec}$. With slight variations from token to token, stimulus level measured with an SLM (B\&K type 2204) set on the "fast" meter response was approximately $47 \mathrm{dBC}$ for the broadband bursts and $42 \mathrm{dBC}$ for the high- and low-passed bursts.

\section{Procedure}

Six different stimulus conditions were constructed from the various noise bursts. As shown in Table 1, the leading stimulus for both the train and the test burst pairs was always a broadband noise burst (B), and the lagging stimulus could be ether the low-pass (L) or the high-pass $(\mathrm{H})$ filtered burst. On three of the six conditions, the test burst pair consisted of a broadband lead and a low-frequency echo (BL). This test burst pair was (1) presented in isolation (the "no conditioning train," or NCBL condition), (2) preceded by a conditioning train that also had a broadband lead and a low-frequency emphasis echo (BL-BL), and (3) preceded by a train that had a broadband lead and a high-frequency emphasis echo $(\mathrm{BH}-\mathrm{BL})$. On the remaining three conditions, the test burst pair had a broadband lead and a high- 
Table 1

Experimental Conditions

\begin{tabular}{lccccc}
\hline & \multicolumn{2}{c}{ Train } & & \multicolumn{2}{c}{ Test } \\
Condition & Lead & Lag & & Lead & Lag \\
\hline NCBL & & & & broadband & low-pass \\
BL-BL & broadband & low-pass & & broadband & low-pass \\
BH-BL & broadband & high-pass & & broadband & low-pass \\
NCBH & & & & broadband & high-pass \\
BH-BH & broadband & high-pass & & broadband & high-pass \\
BL-BH & broadband & low-pass & & broadband & high-pass \\
\hline
\end{tabular}

frequency echo $(\mathrm{BH})$, and it was (1) presented in isolation (NCBH), (2) preceded by a train of similar burst pairs $(\mathrm{BH}-\mathrm{BH})$, or (3) preceded by a train of different burst pairs $(\mathrm{BL}-\mathrm{BH})$. During the test burst pair, the leading stimulus was always presented from one of the loudspeakers at $42^{\circ}$ or $48^{\circ}$ left of midline (varied randomly), and the lagging stimulus was presented from one of the loudspeakers at $35^{\circ}$ or $55^{\circ}$ right of midline. During the conditioning train (BL-BL, $\mathrm{BH}-\mathrm{BL}, \mathrm{BH}-\mathrm{BH}$, and $\mathrm{BL}-\mathrm{BH}$ conditions), the leading stimulus was presented from $42^{\circ}$ or $48^{\circ}$ left of midline (held constant throughout the train but varied randomly from trial to trial), and the lagging stimulus was presented from the loudspeaker at $45^{\circ}$ right of midline and was then switched to $35^{\circ}$ or $55^{\circ}$ right on the test burst pair. Separate randomizations were used to locate the leading stimulus during the train and the test, so that, on half of the trials, the location of the lead switched between $42^{\circ}$ and $48^{\circ}$ between the conditioning train and the test burst. The purpose of this procedure was to minimize the subjects' reliance on directional shifts in the fused auditory image, forcing them to localize the shift in direction of the lagging sound.

The subjects were instructed to face directly ahead, and their task was to report, by pressing the appropriate key on a response box held on the lap, which of the two loudspeakers at $35^{\circ}$ or $55^{\circ}$ right delivered the lagging stimulus on the test pair. On half of the trials, the lag was presented from the loudspeaker at $35^{\circ}$; on the other half, it was presented from $55^{\circ}$. The correct location of this delayed burst was shuffled, such that random guessing would produce chance performance of $50 \%$. The subjects were given correct-answer feedback on every trial by the illumination of the appropriate light on the response box. Performance on this objective discrimination task has been shown to be highly correlated with echo thresholds measured by a subjective method in which listeners reported whether or not they heard an echo on each trial (Freyman, Clifton, \& Litovsky, 1991, Experiment 3 ). In the present task, echo threshold was specified in terms of localization, because the subjects had to make a directional discrimination of the lagging sound as a separate audible event.

Each condition was presented in a run of 30 trials, during which the delay between the lead and lag bursts was held constant. A range of four test delays was chosen for each subject using the following tracking method. The subjects first listened to a block of the six stimulus conditions at a long lead-lag delay $(15-20 \mathrm{msec})$ on which the lagging burst was clearly audible, to ensure that they could reliably localize the lagging sound. Once they could do so, the block of six conditions was presented again using a lead-lag delay that was 2 msec lower than the initial block, followed by successive blocks that decremented the lead-lag delay by 2 msec each time. Testing continued in this manner until a lead-lag delay was reached at which the subjects' performance dropped to near chance $(50 \%)$ on all six conditions. The four shortest delays were chosen as the subject's test burst delays, and the block of six conditions was presented two more times at each of those four delays. Thus, for each of the six stimulus conditions, data were collected for a total of three 30-trial runs (a total of 90 trials) at each of four test delays. A blocked design was employed such that all six experimental conditions were presented at a given lead-lag delay before proceeding to a new delay. The order of presentation of the six conditions within each block was shuffled.

\section{RESULTS}

Discrimination performance differed depending on whether the test burst echo had a low-frequency emphasis (NCBL, BL-BL, and BH-BL conditions) or a highfrequency emphasis $\mathrm{NCBH}, \mathrm{BH}-\mathrm{BH}$, and $\mathrm{BL}-\mathrm{BH}$ conditions), so results are presented separately for each frequency condition.

Individual results for all conditions with a low-frequency test burst echo are displayed in Figure 3, in which discrimination performance is plotted as a function of lead-lag delay. Figure 4 presents the mean discrimination performance for the three conditions. Of the three conditions on which the test burst echo had a low-frequency emphasis, discrimination performance was poorest when the BL test burst pair was preceded by a train of identical test burst pairs (BL-BL condition). These data reflect the buildup of echo suppression that occurred when the subjects heard a redundant sound preceding the test burst pair, relative to discrimination of the test burst pair in isolation (NCBL). In contrast, when the lag during the train had a high-frequency emphasis and then suddenly switched to a low-frequency lag on the test burst pair (the BH-BL condition), performance was as good as - and, for some subjects, better than - that for the isolated test burst pair. In other words, a high-frequency echo during the conditioning train had little effect on the discriminability of the low-frequency echo during the test burst.

A 3 (condition) $\times 4$ (delay) analysis of variance (ANOVA) of the $d^{\prime}$ scores revealed significant main effects of delay $[F(3,15)=55.78, p<.001]$ and condition $[F(2,10)=$ $32.99, p<.001]$ and a delay $\times$ condition interaction $[F(6,30)=5.49, p<.001]$. Follow-up comparisons were conducted to examine the main effect of condition. The $\mathrm{BL}-\mathrm{BL}$ condition had lower discrimination performance than the NCBL condition $[F(1,5)=29.76, p<.01]$ and the $\mathrm{BH}-\mathrm{BL}$ condition $[F(1,5)=52.46, p<.001]$. The latter comparison suggests that any buildup of echo suppression that occurred during the burst train was disrupted by a change in the frequency content of the echo from train to test. The delay $X$ condition interaction was due to the lack of any significant differences between the conditions at the shortest delay tested, with the subjects' performance near chance on all three conditions.

The pattern of results was different for the three conditions on which the test burst echo had a high-frequency emphasis ( $\mathrm{NCBH}, \mathrm{BH}-\mathrm{BH}$, and $\mathrm{BL}-\mathrm{BH})$. The subjects had great difficulty discriminating the location of the high-frequency echo when the test burst pair was presented in isolation (NCBH), even at the longest lead-lag delays. This difficulty in discriminating the high-frequency echo prevented meaningful comparisons among the three conditions. Figure 5 presents the mean discrimination performance over all subjects. Essentially flat curves are evident for the three conditions, with $d^{\prime}$ scores below 1.0 across all delays. In sum, performance on the NCBH condition was too poor to measure buildup of echo suppression during the $\mathrm{BH}-\mathrm{BH}$ train, making it impossible to determine 


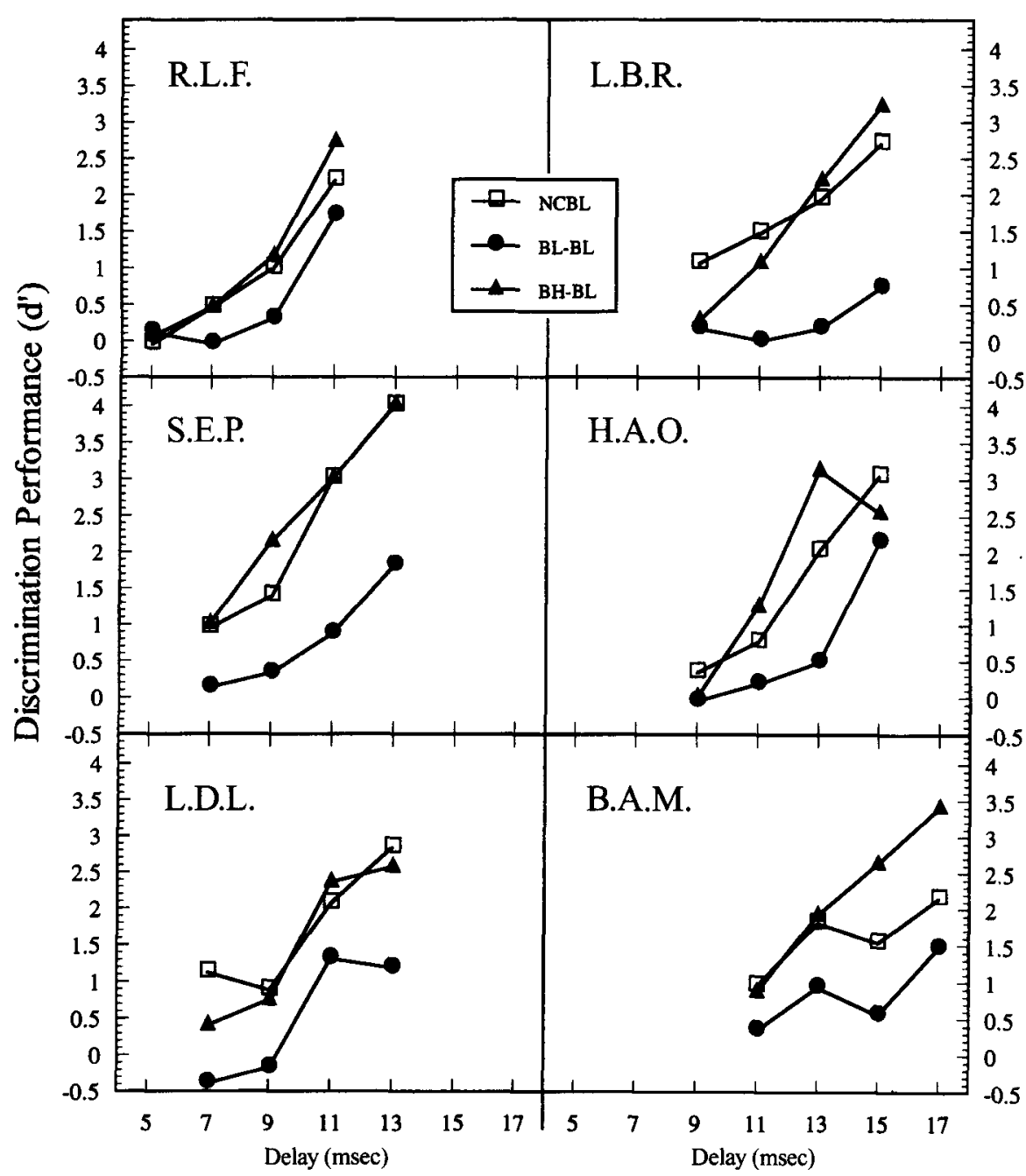

Figure 3. Individual subject discrimination performance for conditions on which the lagging sound in the test burst was low-frequency emphasis.

whether changing the echo's spectrum from train to test (BL-BH condition) affected discrimination of the highfrequency echo during the test burst pair.

We reasoned that the subjects' difficulty in localizing the high-frequency echo, even when it was presented in isolation (NCBH), was due to the fact that it may have been less audible than the low-frequency echo. The range of test delays chosen for each subject in our screening procedure was likely below echo threshold for the high-frequency echoes, although appropriate for low-frequency echoes. We retested 5 subjects and attempted to enhance the audibility of the high-frequency echo either by increasing the delay above echo threshold (to $23 \mathrm{msec}$ ) or by increasing the overall intensity of the burst pairs by $5 \mathrm{~dB}$ (thus lowering echo threshold; see Babkoff \& Sutton, 1966). We used one of these two methods with each subject in order to find one condition on which the subjects could reliably discriminate (with a $d^{\prime}$ of 1.5 or greater) the location of the test echo on the NCBH condition. One subject (H.A.O.) did not need to be retested, because she could discriminate the echo fairly well at the longest of her initial four test delays (15 msec), at a $d^{\prime}$ of 1.4. Subjects S.E.P. and L.B.R. showed improved discrimination performance on the $\mathrm{NCBH}$ condition when tested at the longer delay $(23 \mathrm{msec})$. Subjects R.L.F. and L.D.L., on the other hand, showed improved discrimination performance when tested at the higher intensity, using the longest delay of their initial set of four. Neither approach enabled Subject B.A.M. to localize the high-frequency echo. A full set of data ( 90 trials at each of the three conditions) was then collected for the 4 subjects (S.E.P., L.B.R., R.L.F., and L.D.L.) who could now localize the high-frequency emphasis echo on the NCBH condition. Their data, as well as H.A.O.'s original data, are presented in Figure 6. For each of the 5 subjects, discrimination of the echo was poorest on the BH-BH condition, when the frequency of the lag was held constant from train to test, indicating the buildup of echo suppression that occurred during the train. When the spectrum of the 


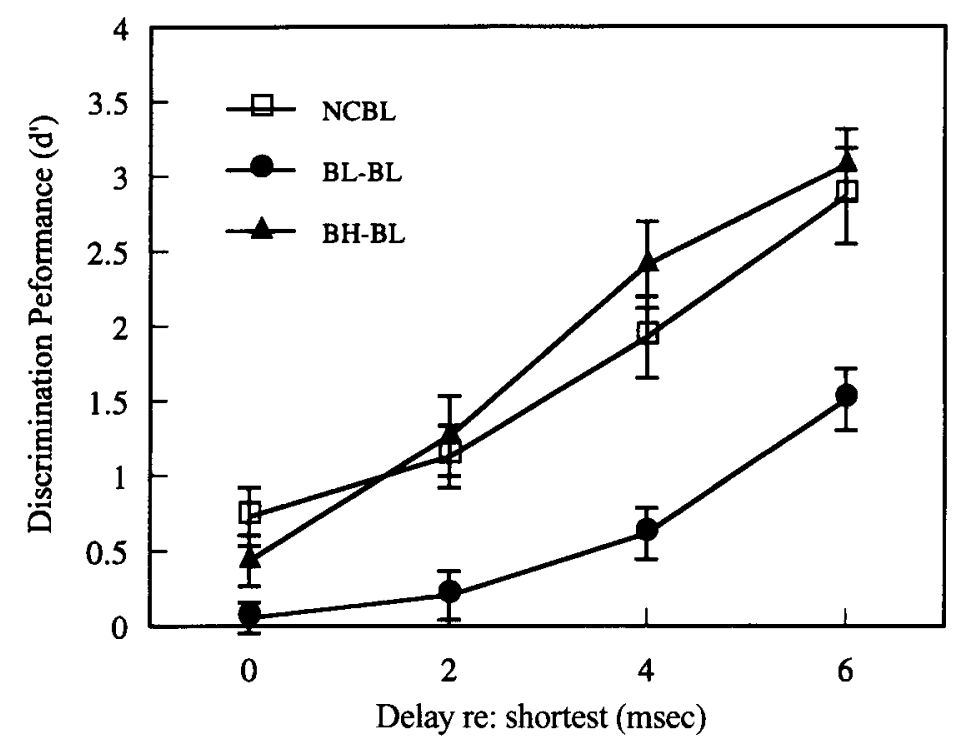

Figure 4. Average discrimination performance for conditions on which the lagging sound in the test burst was low-frequency emphasis. Because a different range of lead-lag delays was selected to span the psychometric function for each subject (see Figure 3), the values along the abscissa indicate the delay relative to the shortest delay used for each subject. Error bars indicate the standard error of the mean.

echo switched after the train (BL-BH condition), discrimination performance improved. An ANOVA on the $d$ 'scores revealed a significant main effect of condition $[F(2,8)=$ $19.26, p<.01]$, which was due to significant differences between discrimination performance on the $\mathrm{NCBH}$ and $\mathrm{BH}-\mathrm{BH}$ conditions $[t(4)=4.73, p<.01]$, and improved discrimination performance on the $\mathrm{BL}-\mathrm{BH}$ condition relative to the $\mathrm{BH}-\mathrm{BH}$ condition $[t(4)=4.10, p<.01]$.

\section{DISCUSSION}

The purpose of the present study was to test the hypothesis that echoes that are environmentally improbable cause a breakdown of an adapted echo threshold. We hypothesized that, during a redundant train of information with a leading broadband noise burst and a lagging burst that was either low- or high-pass filtered, subjects would develop expectations about the echoic properties of a simulated acoustic environment. Such expectations can raise echo threshold by several milliseconds, resulting in better echo suppression. When, on the test burst pair, these expectations were violated in ways that indicated an improbable occurrence in the environment (in this case, a sudden change in the absorptive qualities of the reflective surface), we hypothesized that the built-up echo suppression would be disrupted. The results were as predicted. When the spectral content of the test burst pair was identical to that of the train (BL-BL and $\mathrm{BH}-\mathrm{BH})$ discrimination performance deteriorated, relative to when the test burst pair was presented in isolation. When the frequency content of the lagging burst changed between train and test from high-frequency emphasis to low-frequency emphasis (BH-BL condition) the buildup in echo suppression was disrupted by the new echo, and the subjects could discriminate the location of the lagging burst. The same effect was seen, to a large extent, when the lagging burst changed from a low-frequency emphasis to a high-frequency emphasis between train and test (BL-BH condition). A frequency change such as the one simulated here, in which the leading sound source is held constant but the spectral composition of the echo changes, is unlikely to occur naturally, because it would indicate that the absorption characteristics of the reflective surface had abruptly changed. Both of our test burst pairs simulated echoes that could occur in natural environments. The low-frequency echo would be typical of the filtering done by heavy carpeting or an audience seated in heavily upholstered chairs. Both of these surfaces absorb frequencies higher than $1-2 \mathrm{kHz}$ more efficiently than they absorb lower frequencies. Our high-frequency echo would be typical of reflections filtered by a glass window or thin plywood mounted with airspace behind it; these surfaces reflect high frequencies more efficiently than they reflect low frequencies (see Figures 3.2 and 3.3 in Kinsler, Frey, Coppens, \& Sanders, 1982 , for displays of the absorption properties of these and a variety of other materials). An environmental situation comparable to the acoustic situation simulated by the $\mathrm{BH}-\mathrm{BL}$ condition would be if a glass window or wooden paneling (both of which reflect high frequencies quite well) suddenly was covered with heavy carpeting (reflecting low frequencies while absorbing highs; Knudsen \& Harris, 1978, pp. 102-104, 377). Our hypothesis is that 


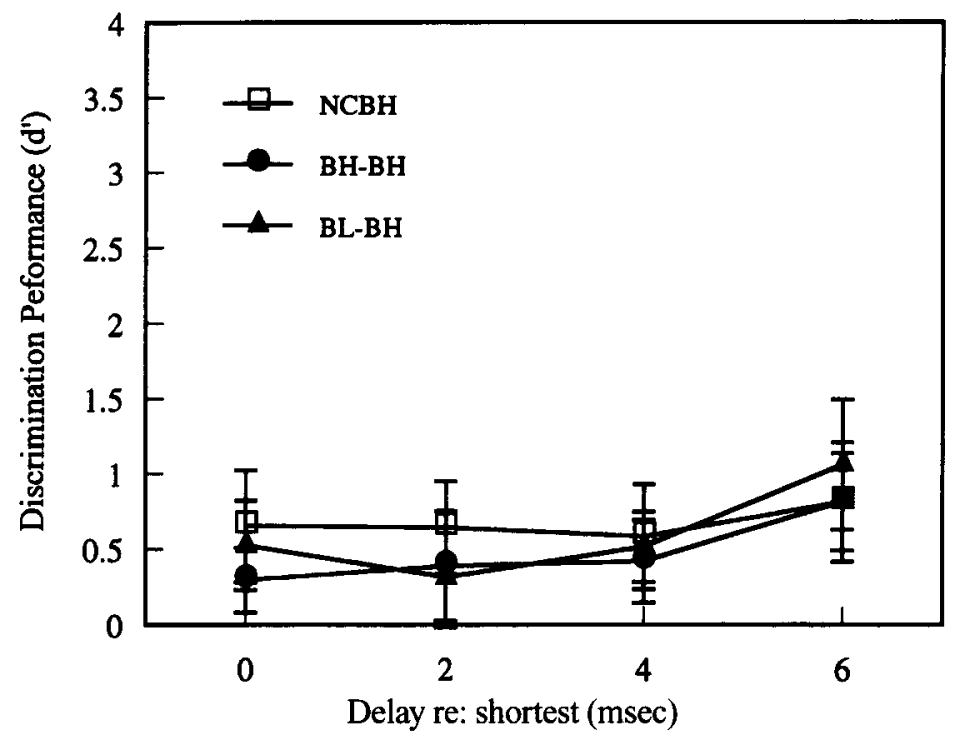

\begin{abstract}
Figure 5. Average discrimination performance for conditions on which the lagging sound during the test burst was high-frequency emphasis. Because a different range of lead-lag delays was selected to span the psychometric function for each subject, the values along the abscissa indicate the delay relative to the shortest delay used for each subject. Error bars indicate the standard error of the mean.
\end{abstract}

this sudden change results in a reexamination of the test burst pair (which entails a lowering of echo threshold), because such a change in room acoustics is unlikely to occur in the listeners' experience. A role for experience in sound localization was also put forward by Plenge (1974). In his article on the difference between localization and lateralization, Plenge emphasized that our ability to localize sound was based on long-term learning beginning in childhood. When we enter a room, we are able to calibrate input against stored knowledge, updating that knowledge each time we come into the room. For example, a room full of people will not have the absorption properties of that same room without people, as designers of auditoria well know. The present study shows that listeners not only encode this information contained in echoes but also respond to an unexpected change in reflected sound.

When the test burst echo had a low-frequency emphasis, the data conformed to the hypothesis quite well. The results for conditions with the high-frequency echo were not so straightforward. The flat lines and poor performance for all of the high-frequency echo conditions in Figure 5 indicate that the subjects either did not hear the test burst echo at all or, if heard, were unable to localize it. Let us examine each of these possibilities. Did the subjects fail to hear the high-frequency echo because the broadband lead dominated it? A similar asymmetry in the precedence effect across frequency has been reported elsewhere. Using narrow-band noise bursts, Blauert and Divenyi (1988), Divenyi (1992), and Shinn-Cunningham, Zurek, Durlach, and Clifton (1995) found that leading low frequencies suppress lagging high frequencies better than high frequencies suppress lows. Our broadband lead contained low frequencies that may have dominated the high-frequency echo to a greater extent than it dominated the low-frequency echo, rendering the former less audible.

The second issue is difficulty in localization. Remember that our task required the subjects to discriminate the location of the echo. The spectrum of the high-frequency echo contains most of its energy between 2 and $8 \mathrm{kHz}$. Minimal audible angle (MAA) rises steeply for this frequency range, especially for sound sources off-midline at $30^{\circ}$ and beyond (Mills, 1972). The MAA is virtually offscale at $60^{\circ}$. Our echo loudspeakers were located at $35^{\circ}$ and $55^{\circ}$, which is distinctly in the range of positions that might impair listeners' localization. Mills used tones, and we used stimuli with a broader spectrum; however, the same frequency effects were probably at work in our situation. It is likely that our subjects' poor performance with the highfrequency lag burst was due both to the difficulty in localizing it and to its lower audibility due to dominance of lower frequencies in the leading broadband sound over the higher frequencies in the lagging sound. After we made the echo more audible by either increasing the delay between lead and lag or raising the overall SPL, the subjects' discrimination performance did follow the predicted pattern: decreasing when the echo's frequency content was held constant from train to test (BH-BH), and returning toward unadapted levels when the echo frequency changed from train to test (BL-BH).

The fact that echo thresholds were different for highand low-frequency echoes introduced a minor complication into our original symmetrical design. We had planned to use the same range of delays in all series, and our screening procedure led us to set these delays for the lower thresh- 


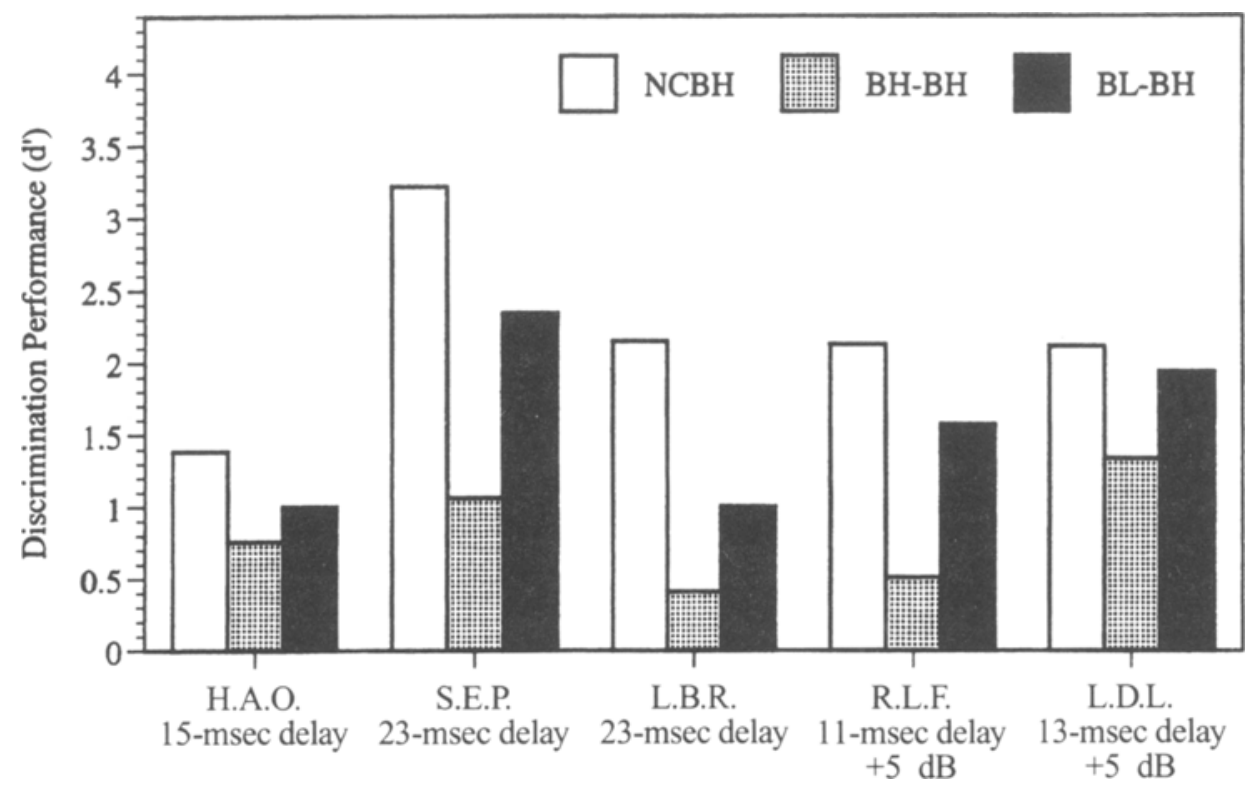

Figure 6. Individual subject discrimination performance for conditions on which the lagging sound during the test burst was high-frequency emphasis, after the subjects had been retested using either a longer lead-lag delay (S.E.P. and L.B.R.) or a higher intensity stimulus (R.L.F. and L.D.L.). H.A.O. did not need to be retested, since she did not have as much difficulty as the other subjects in hearing the high-frequency echo on the test burst; her original data are presented here.

old, which was appropriate only for low-frequency echoes. This necessity to change the delay for the high-frequency echo highlights a previous observation-namely, that the shift in echo perceptibility that occurs during repeated stimulation is a near-threshold phenomenon (Clifton et al., 1994). Its temporal limits are centered around the echo threshold of the isolated lead-lag pair. The delay of the test burst pair must be near this threshold in order to measure the loss in perceptibility after a repeating train or, in the case of the present study, increased perceptibility after an improbable change.

The present results lend further support to the hypothesis that echo suppression is a dynamic process, subject to listeners' expectations about what "reasonable" echoes should be. Sudden changes in the simulated acoustic environment that reflect improbable real-world events result in increased audibility/discriminability of echoes or a lowering of echo threshold. Such changes include (1) suddenly switching from a single-source stimulus to one that has an echo (Freyman et al., 1991), (2) suddenly switching the spatial locations of the leading and lagging sounds (Blauert \& Col, 1992; Clifton, 1987), (3) suddenly increasing or decreasing the delay between the lead and lag (Clifton et al., 1994), and (4) suddenly changing the frequency relationship between the lead and lag (the present results). In contrast, sudden changes that reflect probable environmental events have no effect on echo threshold. Clifton et al.'s (1994) Experiment 2 is of particular relevance here because a change in frequency between train and test burst pairs was predicted not to affect echo threshold. Listeners heard a train of low-frequency lead and lag pairs, followed by a test burst pair in which both lead and lag sounds changed to a higher frequency. Likewise, they heard a train of highfrequency lead and lag pairs, followed by a test burst pair in which both lead and lag sounds changed to a lower frequency. Because both lead and lag sounds changed together, we predicted that the buildup in echo threshold during the train would not be disrupted, and it was not. Such variation in frequency simply indicates to the listener that the frequency of the source has changed, and the echo undergoes a corresponding change. There is nothing unusual in this - most sound sources and their respective echoes are constantly fluctuating in frequency and intensity. Another instance of a change that did not disrupt echo threshold was a condition in which the spatial positions of the leading and lagging sounds shifted simultaneously (Yost \& Guzman, 1995, 1996). A variation in the situation in which the spatial locations of leading and lagging stimuli are rapidly switched led to interesting results. Blauert and Col (1992) found that, if the leading and lagging stimuli are repeatedly alternated, not just switched once as in Clifton (1987), subjects will eventually adapt to the switch, and echo threshold will be maintained. While such an occurrence is relatively unlikely to occur naturally, the effect of repeating the switch several times allows the listener to build up an expectation for its occurrence.

The precedence effect results in the suppression of the location of reflected sounds in the environment and thus enables listeners to accurately localize sound sources within echoic environments. The above data suggest that, although listeners may suppress directional information about echoes, they remain quite sensitive to information carried 
in reflections that informs them about the acoustic properties of surrounding space. Their expectations about this information are based on ongoing acoustic stimulation and past experience. These expectations are revealed when a sudden violation causes a drop in echo threshold. Models of the precedence effect that rely on peripheral adaptation cannot explain the phenomena described in this report and others reviewed above. The data make a strong case for cognitive influences on our perception of echoes.

\section{REFERENCES}

BABKOFF, H., \& SutTon, S. (1966). End point of lateralization for dichotic clicks. Journal of the Acoustical Society of America, 41, 87-102. Blauert, J. (1983). Spatial hearing. Cambridge, MA: MIT Press.

BLAUERT, J. (1996). Spatial hearing: The psychophysics of human sound localization (rev. ed.). Cambridge, MA: MIT Press.

Blauert, J., \& Col, J. P. (1992). Irregularities in the precedence effect. In Y. Cazals, K. Horner, \& L. Demany (Eds.), Auditory physiology and perception (pp. 531-538). Oxford: Pergamon.

Blauert, J., \& Divenyi, P. L. (1988). Spectral selectivity in binaural contralateral inhibition. Acustica, 66, 267-274.

CLIfTON, R. K. (1987). Breakdown of echo suppression in the precedence effect. Journal of the Acoustical Society of America, 82, 1834-1835.

Clifton, R. K., \& Freyman, R. L. (1989). Effect of click rate and delay on breakdown of the precedence effect. Perception \& Psychophysics, 46, 139-145.

Clifton, R. K., \& Freyman, R. L. (1997). The precedence effect: Beyond echo suppression. In R. H. Gilkey \& T. R. Anderson (Eds.), Binaural and spatial hearing in real and virtual environments (pp. 233255). Mahwah, NJ: Erlbaum.

Clifton, R. K., Freyman, R. L., Litovsky, R. Y., \& McCall, D. D. (1994). Listeners' expectations about echoes can raise or lower echo threshold. Journal of the Acoustical Society of America, 95, 1525-1533.

DiVENYI, P. L. (1992). Binaural suppression of nonechoes. Journal of the Acoustical Society of America, 91, 1078-1084.

Freyman, R. L., Clifton, R. K., \& Litovsky, R. Y. (1991), Dynamic processes in the precedence effect. Journal of the Acoustical Society of America, 90, 874-884.

Freyman, R. L., Clifton, R. K., \& McCall, D. D. (1994). Sudden changes in simulated room acoustics influence echo suppression. Journal of the Acoustical Society of America, 97(5, Pt. 2).

HAFTER, E. R., Buell, T. N., \& Richards, V. M. (1988). Onset-coding in lateralization: Its form, site, and function. In G. M. Edelman, W. E Gall, \& W. M. Cowan (Eds.), Auditory function. Neurobiological bases of hearing (pp. 647-676). New York: Wiley.

HARTMANN, W. M. (1997). Listening in a room and the precedence effect. In R. H. Gilkey \& T. B. Anderson (Eds.), Binaural and spatial hearing in real and virtual environments (pp. 191-210). Mahwah, NJ: Erlbaum.

Kinsler, L. E., Frey, A. R., Coppens, A. B., \& SAnders, J. V. (1982). Fundamentals of acoustics (3rd ed.). New York: Wiley.

KNUdSEN, V. O., \& HARRIS, C. M. (1978). Acoustical designing in architecture. New York: American Institute of Physics.

Mrlls, A. W. (1972). Auditory localization. In J. V. Tobias (Ed.), Foundations of modern auditory theory: Vol. II (pp. 301-348). New York: Academic Press.

Plenge, G. (1974). On the difference between localization and lateralization. Journal of the Acoustical Society of America, 56, 944-951.

Shinn-Cunningham, B. G., Zurek, P. M., Durlach, N. I., \& Clifton, R. K. (1995). Cross-frequency interactions in the precedence effect. Journal of the Acoustical Society of America, 98, 164-171.

Thurlow, W. R., \& Parks, T. E. (1961). Precedence-suppression effects for two click sources. Perceptual \& Motor Skills, 13, 7-12.

Wallach, H., Newman, E. B., \& Rosenzweig, M. R. (1949). The precedence effect in sound localization. American Journal of Psychology, 62, 315-336.

Yost, W. A., \& Guzman, S. J. (1995). Precedence and plausibility. Journal of the Acoustical Society of America, 97(5, Pt. 2), 3412.

Yost, W. A., \& Guzman, S. J. (1996). Auditory processing of sound sources: Is there an echo in here? Current Directions in Psychological Science, 5, 125-131.

ZUREK, P. M. (1987). The precedence effect. In W. A. Yost \& G. Gourevitch (Eds.), Directional hearing (pp. 85-105). New York: Springer-Verlag.

(Manuscript received August 19, 1996; revision accepted for publication April 2, 1997.) 